\title{
Flora asociada y perspectivas de conservación para una población de Beilschmiedia berteroana (Gay) Kosterm. (Lauraceae) en un sistema agrícola de Chile central
}

\section{Associated flora and conservation prospects for a Beilschmiedia berteroana (Gay) Kosterm. (Lauraceae) population in an agroecosystem of Central Chile}

\author{
César A. Sepúlveda ${ }^{1 *}$, Patricio Peñailillo² \& David H. Boshier ${ }^{3}$ \\ 1Departamento de Fiscalización y Evaluación Ambiental, Corporación Nacional Forestal, Región del Maule. 4 norte 1673 , \\ Talca, Chile. \\ ${ }^{2}$ Instituto de Ciencias Biológicas, Universidad de Talca. 2 Norte 685, Talca, Chile. \\ ${ }^{3}$ Department of Plant Sciences, University of Oxford. South Parks Road, Oxford OX13RB, UK. \\ "cesar.sepulveda@conaf.cl
}

\begin{abstract}
RESUMEN
Beilschmiedia berteroana (Lauraceae) es un árbol endémico y en peligro de extinción, escasamente distribuido desde

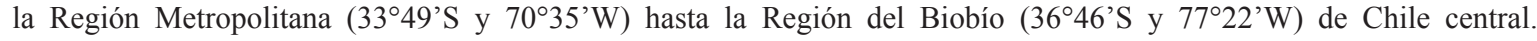
Históricamente, su área de ocupación ha mantenido una prolongada e intensa actividad silvoagropecuaria y concentra el $23 \%$ de las plantas amenazadas del país. Esta situación implicaría revisar y ajustar las estrategias o métodos de conservación para este tipo de paisaje. El trabajo tiene por objetivo contribuir al conocimiento y conservación de una población de $B$. berteroana inserta en un sistema agrícola y ubicada en la Depresión Intermedia de Chile central. Para ello se propone: a) describir demográficamente la población de B. berteroana y flora vascular asociada y b) examinar las posibilidades de su conservación en un sistema agrícola. Se aplicó un censo a B. berteroana y un Sondeo Botánico Rápido (SBR) a la flora asociada. El censo detectó 37 individuos de $B$. berteroana con un total de 647 rebrotes, distribuyéndose el 13,5\% en forma aislada y el 86,5\% en pequeños grupos. El SBR reveló 27 especies de plantas vasculares, predominando los arbustos (37,0\%), seguido por los árboles $(33,3 \%)$, hierbas perennes $(18,5 \%)$ y trepadoras leñosas $(11,1 \%)$. El tipo ecológico de las especies se distribuye por el tipo mesófilo (55,6\%), seguido por el esclerófilo (25,9\%) e higrófilo (18,5\%). Los mayores valores de importancia recaen en las especies nativas Colliguaja dombeyana $(24,22)$ y Salix humboldtiana $(17,61)$, seguido por la especie introducida Rubus ulmifolius (16,59). De acuerdo con el origen, el 51,9\% de las especies son nativas, 29,6\% endémicas y 18,5\% introducidas. Adicionalmente, se informa el hallazgo de Tarasa reichei, un arbusto poco conocido y endémico de Chile central. La nueva localidad (Longaví) para T. reichei extiende el límite sur de la especie en $77 \mathrm{~km}$ y la desplaza por vez primera a la Depresión Intermedia. Se revisan las posibilidades de conservación para B. berteroana y $T$. reichei y se propone la conservación circa situm como método complementario.
\end{abstract}

Palabras clave: Especies amenazadas, conservación circa situm, sistemas agrícolas.

\begin{abstract}
Beilschmiedia berteroana (Lauraceae) is an endangered tree, sparsely distributed and endemic from the Metropolitan Region ( $33^{\circ} 49^{\prime} \mathrm{S}$ and $\left.70^{\circ} 35^{\prime} \mathrm{W}\right)$ to the Biobío Region $\left(36^{\circ} 46^{\prime} \mathrm{S}\right.$ and $\left.77^{\circ} 22^{\prime} \mathrm{W}\right)$ of central Chile. Historically, the area of its occurrence has been subject to prolonged and intense agricultural activity and harbours $23 \%$ of the country's threatened plant species. This situation suggests the need to revise and adjust conservation strategies and approaches for this type of landscape. The objective of the study is to contribute to the knowledge and conservation of a population of $B$. berteroana found within an agroecosystem in the central valley of Chile, a) describing a population of $B$. berteroana and any associated vascular flora, and b) revising the possibilities of conservation in an agroecosystem. A census was applied to B. berteroana and a Rapid Botanical Survey (RBS) to the associated flora. The census detected 37 individuals of $B$. berteroana with a total of 647 coppice shoots, with $13.5 \%$ of individual found in isolated form and $86.5 \%$ in small groups. The RBS found 27 species of vascular plants, predominantly shrubs $(37.0 \%)$, followed by trees $(33.3 \%)$, perennial herbs $(18.5 \%)$ and lianas (11.1\%). The ecological types of species where mesophilic (55.6\%), followed by sclerophyllous (25.9\%) and hygrophyllous (18.5\%) types. The highest Importance Values are associated with the native species Colliguaja dombeyana (24.22) and Salix humboldtiana (17.61), followed by the introduced Rubus ulmifolius (16.59). By origin, 51.9\% of the
\end{abstract}


species are native, endemic are $29.6 \%$ and $18.5 \%$ are introduced plants. In addition, the finding of Tarasa reichei, a little known endemic shrub of Chile central, is reported. The new locality (Longaví) extends the southern boundary of $T$. reichei by $77 \mathrm{~km}$, moving it to the central valley. We review conservation possibilities for $B$. berteroana and $T$. reichei and propose that conservation of circa situm as a complementary method.

KEYWORDS: Endangered species, circa situm conservation, agroecosystem.

\section{INTRODUCCIÓN}

Históricamente en la zona de Chile central la expansión de las fronteras agrícolas, forestales y de centros urbanos ha originado un intenso y prolongado cambio en el uso del suelo, considerándose una de las mayores transformaciones en el paisaje de los últimos 200 años (Lara et al.1989, 2012, Henríquez et al. 2006). Para autores como Echeverría et al. (2007) y Aguayo et al. (2009), esta trasformación ha originado que entre las regiones Metropolitana y del Biobío se encuentren amenazados importantes elementos de biodiversidad endémicos de Chile central. Dado que está zona concentra la mayor actividad silvoagropecuaria del país y forma parte de uno de los 25 hotspots de biodiversidad global debido a su alta diversidad, riqueza y endemismo (Myers et al. 2000, Ibarra et al. 2005), es preciso implementar estrategias de conservación capaces de integrar las actividades propias de la producción con aquellas destinadas a la conservación de la biodiversidad, más aún, cuando el $23 \%$ de las plantas amenazadas del país se encuentra en esta zona (MMA 2017).

Un endemismo singular que habita en las condiciones ya mencionadas es Beilschmiedia berteroana (Gay) Kosterm. "Belloto del Centro", una especie de la familia Lauraceae, de crecimiento arbóreo, hojas persistentes y duras. Muñoz (1973) lista a $B$. berteroana como una de las especies chilenas en vías de extinción debido a la fuerte reducción de su hábitat. Luego es catalogada por un comité de expertos en la categoría "En peligro" dada la escasez de sus poblaciones y restringida distribución natural (Benoit 1989). Esto motivó al Ministerio de Agricultura que la declarara Monumento Natural, prohibiendo la corta o eliminación de cualquier ejemplar (Decreto Supremo $\mathrm{N}^{\circ} 13$ de 1995). Sin embargo, en la lista roja de plantas amenazadas de la Unión Internacional para la Conservación de la Naturaleza (UICN) de 1997 fue considerada en la categoría "Rara" (Walter \& Gillett 1998). Con criterios más recientes de la UICN fue propuesta como una especie "En Peligro" al presentar un área de ocupación estimada menor a $500 \mathrm{~km}^{2}$, una distribución severamente fragmentada y restringida a márgenes de ríos o esteros (Hechenleitner et al. 2005). Según la clasificación de especies del Ministerio del Medio Ambiente, la especie en cuestión fue corroborada en la categoría "En Peligro" (Decreto Supremo N`50 de 2008).

El área de distribución natural de $B$. berteroana está restringida a escasos rodales desde la Región Metropolitana
( $33^{\circ} 49^{\prime} \mathrm{S}$ y $70^{\circ} 35^{\prime} \mathrm{W}$ ) hasta la Región del Biobío (36² $46^{\prime} \mathrm{S}$ y $77^{\circ} 22^{\prime} \mathrm{W}$ ), entre los 300 y $1.800 \mathrm{~m}$ de altitud (Cabello et al. 2006). Los bosques de B. berteroana se hallan en la Región del Bosque Caducifolio (Gajardo 1993), ubicándose preferentemente en la precordillera andina, avanzando hacia el Sur por la Depresión Intermedia. Aquí crece sobre suelos fértiles, profundos, generalmente asociados a cursos de agua y lugares de lomajes suaves con pendientes entre 3 y $5 \%$. La vegetación natural acompañante corresponde a especies arbóreas esclerófilas como Cryptocarya alba (Molina) Looser y Peumus boldus Molina y Mirtáceas como Luma apiculata (DC.) Barret, dentro de las especies arbustivas aparecen Aristotelia chilensis (Molina) Stuntz, Colliguaja spp. y Otholobium glandulosum (L.) J.W. Grimes (Cabello et al. 2006).

Aun cuando $B$. berteroana no tiene importancia maderera ni tampoco como leña o carbón, el uso extensivo e intensivo de la tierra para cultivos agrícolas y forestales, ha incidido en la pérdida y eliminación de sus poblaciones (San Martín et al. 2002). De este modo, hoy en día, en su rango de distribución es posible observar individuos aislados o en pequeños grupos fuera de su hábitat natural o insertos en sistemas agrícolas, expresión de la histórica intervención humana a nivel del paisaje. En este sentido, los apuntes de Espinosa (1941) ya indicaban individuos aislados o en pequeñas agrupaciones ubicadas en terrenos húmedos a orillas de cursos de agua en los fundos "Porvenir" y "Huadantún" en el valle del río Putagán, y en la hacienda "La Quinta" de Longaví, donde rodeado de terrenos agrícolas se ubicaban los "Bellotales".

Actualmente B. berteroana es conservada in situ en dos unidades del Sistema Nacional de Áreas Protegidas del Estado (SNASPE): i) Reserva Nacional Bellotos del Melado en la precordillera de Linares en la Región del Maule y ii) Reserva Nacional Roblería del Cobre de Loncha ubicada en la Región del Libertador General B. O'Higgins. En esta última, no tan sólo protege las poblaciones más extensas, sino que también la más al norte y oeste de su distribución (Cabello et al. 2006, Ricci et al. 2007). Asimismo, se ha documentado un convenio de conservación para una población cercana a Bulnes entre su propietario y la Universidad Austral de Chile, que incluye la propagación y protección de la regeneración natural (Hechenleitner et al. 2005). Algunas iniciativas de conservación ex situ son reportadas en parques y jardines botánicos del país (Espinosa 1941, Benoit 1989, Rodríguez et al. 1995). 
Flora y conservación de una población de Beilschmiedia berteroana: SEPÚLVEDA, C. ET AL.

Una propuesta novedosa para la conservación de especies es el método circa situm, empleado en situaciones de árboles aislados o en pequeños grupos en su distribución natural, pero fuera del hábitat natural e insertos en sistemas agrícolas o forestales, tal como sucede con algunas poblaciones de $B$. berteroana. La conservación circa situm ha sido desarrollada como un método complementario de conservación para algunas especies arbóreas de Centroamérica (e.g., Leucaena spp., Cordia alliodora (Ruiz \& Pav.) Oken y Swietenia humilis Zucc.) que han logrado conservarse en sistemas agrícolas -altamente perturbados- por su capacidad de regeneración, manejo maderero y no maderero (Brush 1991, Boshier et al. 2004, Barrance et al. 2003, 2009). Las implicaciones biológicas de la conservación circa situm se relacionan con conservar genotipos no encontrados en áreas protegidas, facilitar el flujo génico, mantener poblaciones mínimamente viables e intermediar a polinizadores $\mathrm{y}$ dispersores de semillas (Lander et al. 2009, Arnold et al. 2010). No obstante, Boshier et al. (2004) plantean limitaciones de su aplicación para especies de crecimiento lento, con baja capacidad de regeneración, polinizadas y dispersadas por vectores especialistas. El presente trabajo tiene por finalidad contribuir al conocimiento y conservación de una población de $B$. berteroana inserta en un sistema agrícola y ubicada en la Depresión Intermedia de Chile central. Para ello se propone: a) describir demográficamente la población de $B$. berteroana y flora vascular asociada y b) examinar las posibilidades de conservación en un sistema agrícola. Estos antecedentes, no tan sólo son un aporte al conocimiento de la flora y estado actual de una población remanente de $B$. berteroana, sino que además podría ser útil para las iniciativas nacionales de planificación destinadas para la recuperación, conservación y gestión de especies de plantas amenazadas en Chile.

\section{MATERIALES Y MÉTODOS}

Durante marzo del año 2016 se realizó un censo poblacional para $B$. berteroana en un sistema agrícola de 15,1 ha, ubicado en la localidad de Longaví, Región del Maule. El sitio de estudio se encuentra aproximadamente a $2 \mathrm{~km}$ al oriente del "Bellotal" informado por Espinosa (1941), y actualmente poblado por la ciudad de Longaví. Para cada presencia aislada o en grupo de $B$. berteroana se registró su ubicación a través de sus coordenadas UTM. A cada individuo físiológico o cepa se contabilizó el número de rebrotes, estado fenológico, altura, diámetro a la altura del pecho (DAP), estado fitosanitario y amenazas. La información florística asociada a cada individuo se levantó mediante un sondeo botánico rápido (SBR) según la metodología descrita por Hawthorne \& Abu-Juam (1995) y comprobada para su uso en Chile (Arnold et al. 2010). Para cada especie se obtuvo el valor de importancia (VI) a partir de su frecuencia y dominancia (Matteucci \& Colma 1982). Los valores de recubrimiento se estimaron según BraunBlanquet utilizando la transformación a valores continuos propuesta por Tüxen-Ellenberg (en Van Der Maarel 1979). El esfuerzo de muestreo se evaluó con la curva acumulación de especies, ajustando el modelo de Clench con los programas EstimateS y Statistica (Jiménez-Valverde \& Hortal 2003).

Los nombres de las especies, familias y origen geográfico fueron consultados en el Catálogo de Plantas Vasculares de Flora del Cono Sur (http://www.darwin.edu.ar). Las formas de vida se determinaron según la clave de Mueller-Dombois \& Ellenberg (1974), los tipos ecológicos siguiendo a San Martín \& Sánchez (1999) y las formas de crecimiento por inspección visual en el sitio de estudio. La condición de especie invasora se obtuvo de Fuentes et al. (2014) y de la base de datos del Laboratorio de Invasiones Biológicas (LIB) de la Universidad de Concepción (http://www.lib. udec.cl). Para el estado de conservación de las especies se revisaron las listas oficialmente decretadas en los procesos de clasificación de especies (MMA 2017).

Se evaluó el grado de amenaza de las especies introducidas según el índice desarrollado por Holm et al. (1979), el cual utiliza cuatro categorías: X para especies que son malezas infrecuentes; $\mathrm{C}$ para malezas comunes; $\mathrm{P}$ para malezas principales; y S para malezas serias. Se utilizó esta misma escala, pero convertidos a números $1,2,3$ y 4, respectivamente. El número más grande indica una mayor tendencia a llegar a ser una especie introducida seria para la flora nativa. La abundancia o recubrimiento se obtuvo según los valores trasformados y el grado de amenaza de la suma de la agresividad y abundancia de cada especie.

Los registros botánicos se transformaron a coordenadas geográficas y fueron mapeados con ArcGis con el apoyo de imágenes aéreas de Google Earth.

Material de estudio: Beilschmiedia berteroana. Chile, Región del Maule, Prov. de Linares, Longaví, 150 m, III2016. Sepúlveda \& Peñailillo s/n (CONC183537). Tarasa reichei. Chile, Región del Maule, Prov. de Linares, Longaví, 150 m, IX-2015. Sepúlveda \& Peñailillo s/n (CONC184024).

\section{RESULTADOS}

Se censaron 37 individuos físiológicos o cepas de $B$. berteroana con un total de 647 rebrotes, distribuyéndose el $13,5 \%$ en forma aislada (Sector 1, Fig. 1) y el 86,5\% en pequeños grupos (Sector 2, Fig. 1). Los rebrotes se agruparon en tres rangos de DAP, en orden decreciente se presentan 459 rebrotes $(70,9 \%)$ con DAP inferiores a $5 \mathrm{~cm}$, 159 rebrotes $(24,6 \%)$ entre 5,1 y $10 \mathrm{~cm}$ y 29 rebrotes $(4,5 \%)$ superiores a $10 \mathrm{~cm}$ (Fig. 2). Los rebrotes de dos individuos, Id. 5 e Id. 30, no pudieron censarse dado su difícil acceso (Fig. 2). 


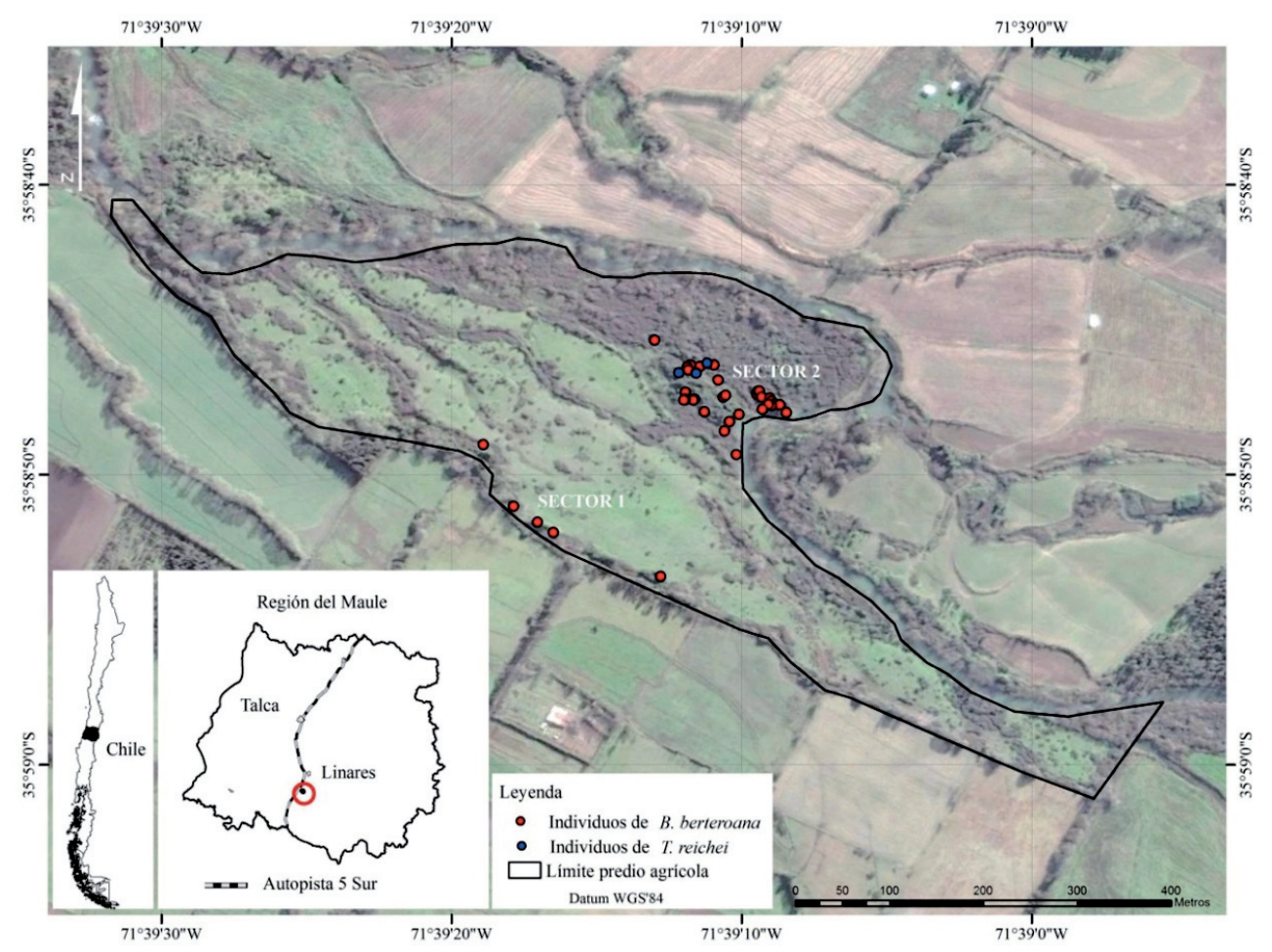

Figura 1. Ubicación de B. berteroana, señalando individuos aislados (sector 1) y en pequeños grupos (sector 2). Además, el Sector 2 es la ubicación de la nueva población de $T$. reichei en la localidad de Longaví. / Location of $B$. berteroana, as isolated individuals (sector 1) and in small groups (sector 2). Sector 2 is also the location of the new population of T. reichei in the locality of Longaví.

Fenológicamente se observaron individuos con restos florales y aproximadamente el 59\% de los individuos presentaban frutos inmaduros. En términos generales su estado fitosanitario es bueno, encontrándose en solo un $3 \%$ de los casos daños provocados por hongos e insectos defoliadores.

El SBR detectó 27 especies de plantas vasculares asociadas a $B$. berteroana (Tabla 1). Las formas de vida predominantes fueron fanerófitos con 22 especies $(81,5 \%)$ y hemicriptófitos con 5 especies $(18,5 \%)$. Los fanerófitos incluyen a las siguientes formas de crecimiento: árboles 9 especies $(33,3 \%)$, arbustos 10 especies $(37,0 \%)$ y trepadoras leñosas o lianas 3 especies $(11,1 \%)$. En cambio, los hemicriptófitos incluyen únicamente a las hierbas perennes con 5 especies $(18,5 \%)$. La distribución ecológica de la flora asociada a $B$. berteroana muestra un predominio de especies mésicas $(55,6 \%)$, seguido por el tipo esclerófilo $(25,9 \%)$ y un nada despreciable porcentaje de especies higrófilas $(18,5 \%)$ (Tabla 1$)$.

En la Tabla 1 se muestran los valores de importancia (VI) para las especies presentes en el sitio. Los mayores valores de importancia se comparten entre especies nativas e introducidas para el estrato arbóreo (especie nativa, Salix humboldtiana Willd. y especie introducida, Salix babylonica L.) y en el estrato arbustivo (especie nativa Colliguaja dombeyana A. Juss. y especie introducida

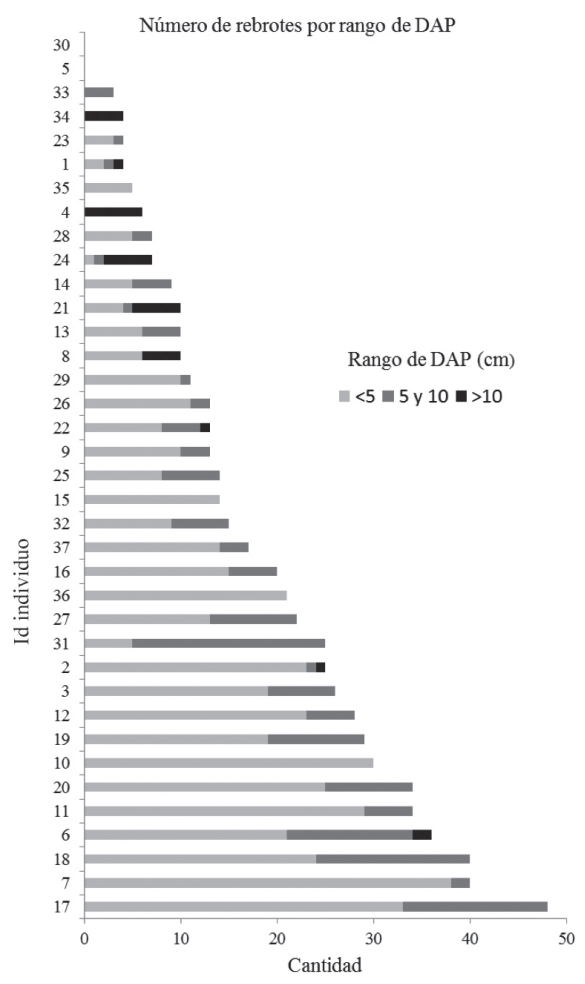

Figura 2. Número de rebrotes por rango de DAP en $B$. berteroana. / Number of resprouts by DBH range in $B$. berteroana. 
Rubus ulmifolius Schott). La hierba introducida Galega officinalis L. es el representante del estrato herbáceo con mayor valor de importancia. Respecto de las especies introducidas consideradas invasoras, la especie con mayor grado de amenaza corresponde a $R$. ulmifolius (zarzamora), principalmente por su agresividad y abundancia. En un grado menor se encuentran las especies herbáceas G. officinalis (galega) y Taraxacum officinale F.H.Wigg. (diente de León), ambas situadas en el borde del sistema agrícola (Tabla 2). De acuerdo con el origen de las especies $14(51,9 \%)$ son nativas, $8(29,6 \%)$ endémicas y $5(18,5 \%)$ introducidas. Dentro de las especies endémicas se registra Tarasa reichei (Phil.) Kaprov. (Tabla 1, Figs. 1 y 3), un arbusto de la familia Malvaceae (Marticorena 2005) endémico de Chile central, redescubierto luego de 100 años en la localidad de "San Pedro de Alcántara" (Marticorena et al. 2007).

\section{DISCUSIÓN}

La distribución del número de rebrotes, según el DAP, confirma la capacidad de sobrevivir que posee $B$. berteroana a través de la regeneración asexuada o vegetativa. Ello indica que la última intervención humana (e.g., habilitación agrícola y quema) no es mayor a 15 años según la relación entre diámetros y edad de los individuos sugerida por Espinosa (1941) y confirmada con antecedentes orales aportados por lugareños.

Según los parámetros de Clench $(\mathrm{n}=9, \mathrm{~s}$ obs $=27$, $\mathrm{a}=12,329$ y $\mathrm{b}=0,359)$ la proporción de especies muestreada se aproxima al $80 \%$, reflejando el condicionamiento de la flora actual a los eventos históricos del sitio, entre ellos: uso ganadero, rotación de cultivos, habilitación agrícola y quema. El espectro biológico sugerido por las formas de vida coincide con el de formaciones boscosas, puesto que dominan los fanerófitos leñosos (árboles y arbustos) por sobre los hemicriptófitos (hierbas perennes). La ausencia de otras formas de vida como criptófitos y terófitos puede explicarse por la época estival en que se realizó el muestreo, ya que las partes aéreas de la planta se secan y persisten los órganos subterráneos para el caso de los primeros y en forma de semillas, para los segundos. También, cabe hacer notar que tanto caméfitos, criptófitos y terófitos son formas de vida escasas en los bosques de $B$. berteroana, no así los fanerófitos y hemicriptófitos que son los elementos más abundantes (Ramírez et al. 2004).

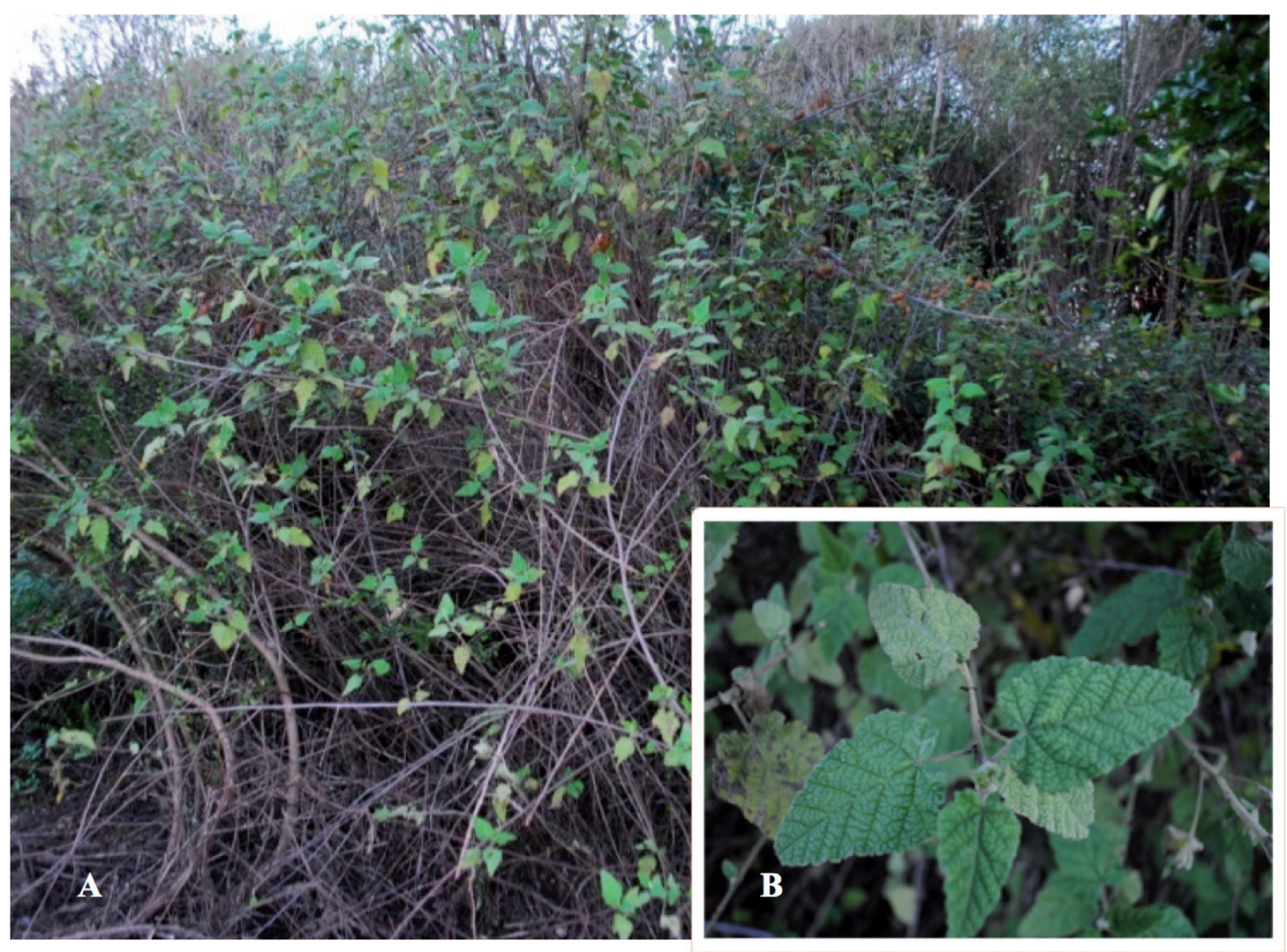

Figura 3. Individuos de Tarasa reichei (A) y detalle de hojas (B). / Individuals of Tarasa reichei (A) and leaf detail (B). 
TABLA 1. Lista de especies presentes en el sitio estudiado, ordenadas por formas de vida, crecimiento y valor de importancia (VI). / List of species present in the study site, ordered by life forms, growth and their importance value (VI).

\begin{tabular}{|c|c|c|c|c|c|c|c|c|c|}
\hline & ESPECIE & FV & $\mathrm{FC}$ & VI & $\mathrm{O}$ & $\mathrm{TE}$ & $\mathrm{EC}$ & $\mathrm{S} 1$ & $\mathrm{~S} 2$ \\
\hline 1 & Salix humboldtiana & $\mathrm{fa}$ & ar & 17,61 & $\mathrm{n}$ & $\mathrm{h}$ & & + & + \\
\hline 2 & Salix babylonica & fa & ar & 13,04 & in & $\mathrm{h}$ & & & + \\
\hline 3 & Maytenus boaria & $\mathrm{fa}$ & ar & 11,26 & $\mathrm{n}$ & $\mathrm{m}$ & & + & + \\
\hline 4 & Beilschmiedia berteroana & $\mathrm{fa}$ & ar & 10,40 & en & $\mathrm{m}$ & $\mathrm{EN}$ & + & + \\
\hline 5 & Aristotelia chilensis & $\mathrm{fa}$ & ar & 6,07 & $\mathrm{n}$ & $\mathrm{e}$ & & + & \\
\hline 6 & Acacia caven & $\mathrm{fa}$ & ar & 4,43 & $\mathrm{n}$ & e & & & + \\
\hline 7 & Myrceugenia exsucca & $\mathrm{fa}$ & ar & 2,79 & $\mathrm{n}$ & $\mathrm{h}$ & & + & \\
\hline 8 & Cryptocarya alba & $\mathrm{fa}$ & ar & 2,21 & en & $\mathrm{e}$ & & & + \\
\hline 9 & Prunus domestica & $\mathrm{fa}$ & ar & 1,16 & in & $\mathrm{m}$ & & & + \\
\hline 10 & Colliguaja dombeyana & $\mathrm{fa}$ & arb & 24,22 & en & $\mathrm{e}$ & & & + \\
\hline 11 & Schinus polygamus & $\mathrm{fa}$ & arb & 12,91 & en & e & & + & + \\
\hline 12 & Azara dentata & $\mathrm{fa}$ & arb & 11,72 & en & $\mathrm{m}$ & & + & + \\
\hline 13 & Otholobium glandulosum & $\mathrm{fa}$ & arb & 8,64 & $\mathrm{n}$ & $\mathrm{h}$ & & + & + \\
\hline 14 & Colliguaja odorifera & $\mathrm{fa}$ & arb & 7,71 & en & $\mathrm{e}$ & & + & \\
\hline 15 & Sophora macrocarpa & $\mathrm{fa}$ & arb & 7,59 & en & $\mathrm{m}$ & & + & + \\
\hline 16 & Myoschilos oblongum & $\mathrm{fa}$ & arb & 2,21 & $\mathrm{n}$ & $\mathrm{m}$ & & & + \\
\hline 17 & Baccharis racemosa & fa & arb & 1,16 & $\mathrm{n}$ & $\mathrm{m}$ & & & + \\
\hline 18 & Cestrum parqui & $\mathrm{fa}$ & arb & 1,16 & $\mathrm{n}$ & $\mathrm{m}$ & & & + \\
\hline 19 & Tarasa reichei & $\mathrm{fa}$ & arb & 1,16 & en & $\mathrm{m}$ & & & + \\
\hline 20 & Rubus ulmifolius & $\mathrm{fa}$ & $\operatorname{tr}$ & 16,59 & in & $\mathrm{m}$ & & + & + \\
\hline 21 & Muehlenbeckia hastulata & $\mathrm{fa}$ & $\operatorname{tr}$ & 11,36 & $\mathrm{n}$ & $\mathrm{e}$ & & + & + \\
\hline 22 & Cissus striata & $\mathrm{fa}$ & $\operatorname{tr}$ & 4,08 & $\mathrm{n}$ & $\mathrm{h}$ & & + & + \\
\hline 23 & Galega officinalis & hc & $\mathrm{hp}$ & 10,52 & in & $\mathrm{m}$ & & + & + \\
\hline 24 & Osmorhiza chilensis & hc & $\mathrm{hp}$ & 4,43 & $\mathrm{n}$ & $\mathrm{m}$ & & + & + \\
\hline 25 & Blechnum hastatum & hc & $\mathrm{hp}$ & 2,21 & $\mathrm{n}$ & $\mathrm{m}$ & $\mathrm{LC}$ & + & \\
\hline 26 & Galium hypocarpium & hc & $\mathrm{hp}$ & 2,21 & $\mathrm{n}$ & $\mathrm{m}$ & & & + \\
\hline 27 & Taraxacum officinale & he & $\mathrm{hp}$ & 1,16 & in & $\mathrm{m}$ & & + & \\
\hline
\end{tabular}

FV: Forma de vida, fa: fanerófitos, hc hemicriptófitos. FC: Forma de crecimiento, ar: árbol, arb: arbusto, hp: hierba perenne, tr: trepadora. VI: Valor de importancia. O: Origen, n: nativa, en: endémica, in: introducida. TE: Tipo ecológico, e: esclerófilo, m: mésico, h: higrófilo. EC: Estado de conservación, EN: en peligro, LC: preocupación menor. S1: Sector 1, S2: Sector 2, +: presencia. / FV: Life form, fa: phanerophytes, hc: hemicryptophytes. FC: Growth form, ar: tree, arb: shrub, hp: perennial herb, tr: lianas. VI: Importance value. O: Origin, n: native, en: endemic, in: introduced. TE: Ecological type, e: sclerophyllous, m: mesophilic, h: hygrophyllous. EC: Conservation status, EN: endangered, LC: least concern. S1: Sector, S2: Sector 2, +: presence.

TABLA 2. Grado de amenaza de las especies introducidas. / Level of threat of introduced species.

\begin{tabular}{cccccc}
\hline Especie & $\begin{array}{c}\text { Clasificación de } \\
\text { Holm }\end{array}$ & $\begin{array}{c}\text { Potencial } \\
\text { INVASOR* }\end{array}$ & Agresividad & Abundancia & $\begin{array}{c}\text { Grado DE } \\
\text { AmENAZA }\end{array}$ \\
\hline Rubus ulmifolius & MMS & 28 (alto) & 4 & 15,56 & 19,56 \\
Galega officinalis & MP & 7 (alto) & 3 & 5,83 & 8,83 \\
Taraxacum officinale & MP & 13 (alto) & 3 & 0,01 & 3,01 \\
\hline
\end{tabular}

MMS: Maleza muy seria. / MMS: Very serious weeds.

MP: Maleza principal. / MP: Principal weeds.

*Fuentes et al. (2014). 
En la población estudiada de $B$. berteroana predominan los arbustos sobre los árboles y el componente herbáceo está formado sólo por hierbas perennes, existiendo una escasez de lianas y enredaderas que sirven de conectores entre los estratos. Esto implica una organización vertical de una vegetación alterada, aunque en rasgos generales coincide con las caracterizaciones fitoclimáticas para áreas templadas (Cain 1950). Desde el punto de vista florístico, no se coincide con las especies descritas para la Región del Bosque Caducifolio (Gajardo 1993), donde su vegetación azonal se asocia a elementos higrófilos (e.g. Laurelia sempervirens Tul., Hydrangea serratifolia (Hook. et Arn.) Phil. f., Luma apiculata, Aextoxicon punctatum Ruiz et Pav., Citronella mucronata (Ruiz et Pav.) D. Don) y a elementos esclerófilos (e.g., Cryptocarya alba, Quillaja saponaria Molina y Retanilla ephedra (Venth.) Brongn.) (Litton et al. 1997, Ramírez et al. 2004). Sin embargo, muestra una mayor similitud a las poblaciones descritas para la Depresión Intermedia, con una flora conformada por especies arbóreas esclerófilas como C. alba y Mirtáceas como L. apiculata (Myrceugenia exsucca (DC.) O. Berg, en este estudio); dentro de las especies arbustivas aparecen A. chilensis, Colliguaja sp. (Colliguaja dombeyana, en este estudio) y Otholobium glandulosum (L.) J.W. Grimes (Cabello et al. 2006.). Por otra parte, al encontrarse $B$. berteroana cercana a un curso de agua (estero Ligual) la acompañan especies propias de una vegetación ribereña como las mencionadas por Troncoso (1988): S. humboldtiana y Maytenus boaria Molina, que en sitios alterados puede incluir especies introducidas como $S$. babylonica, R. ulmifolius y la especie nativa $O$. glandulosum. Esta vegetación es concordante a lo descrito por Espinosa (1941) para un "Bellotal" de la Depresión Intermedia de Chile central.

Los valores obtenidos según el índice de Holm et al. (1979) que evalúan el grado de amenaza de las especies introducidas y consideradas malezas en los sistemas agrícolas (Tabla 2), tales como $R$. ulmifolius (19,56), G. officinalis $(8,83)$ y $T$. officinale $(3,01)$, coinciden con el potencial invasor de las mismas 28 (alto), 7 (alto) y 13 (alto), respectivamente, según los datos de Fuentes et al. (2014) para ecosistemas naturales. El potencial impacto de las especies invasoras como R. ulmifolius es impedir la regeneración de plantas nativas y alterar los mutualismos de polinización, reduciendo el éxito reproductivo de las plantas nativas y aumentando la expansión de polinizadores exóticos (Herrera et al. 2016). G. officinalis es una planta tóxica para los animales (Tapia et al. 2000) y desplaza a las especies nativas en zonas pantanosas muy húmedas (Fuentes et al. 2014). T. officinale es una maleza principal (Matthei 1995) y difícil de erradicar de los cultivos. Aunque para el sitio de estudio su impacto ecológico como planta invasora es bajo, en otras situaciones se comporta con un alto potencial invasor (Herrera et al. 2016).

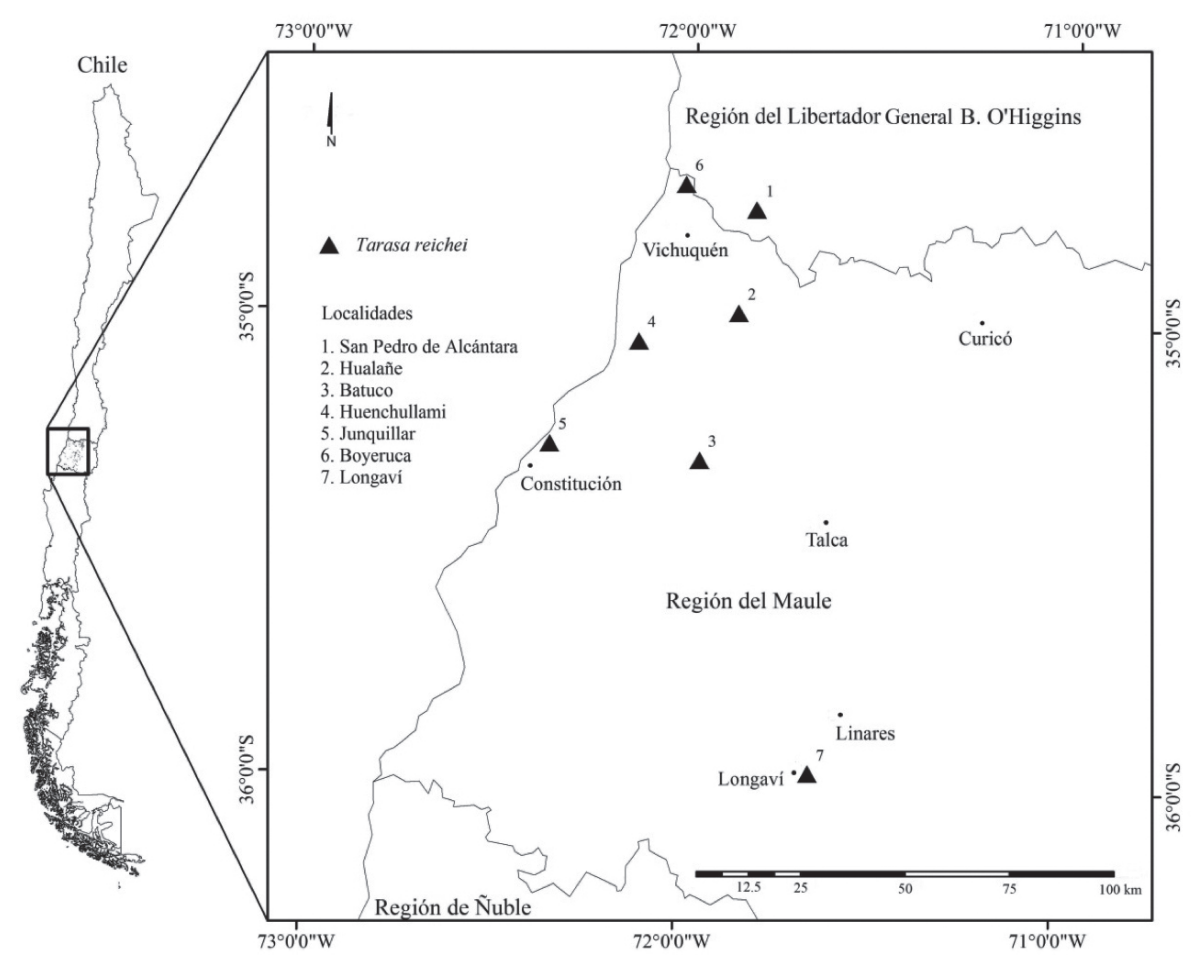

Figura 4. Distribución de Tarasa reichei y nueva localidad (7. Longaví). / Distribution of Tarasa reichei including new location (7. Longaví). 
En relación al hallazgo de $T$. reichei es importante destacar que no tan sólo su límite Sur aumenta en 77 km (Fig. 4) de aquel descrito en un matorral costero de la cuesta de Batuco citado por Gómez et al. (2010), sino que, además, se encuentra por vez primera en la Depresión Intermedia, cercana a cursos de agua e inserta en un sistema agrícola. Las nuevas condiciones de paisaje y hábitat son similares al descrito por Marticorena et al. (2007) para la especie Tarasa umbellata Kaprov., entre ellos: uso agrícola, humedad del suelo, cercanía a un curso de agua y abundancia de la especie invasora $R$. ulmifolius. $T$. reichei no ha sido clasificada aún según los criterios de conservación, pero es un hecho que el paisaje original de Chile central del que forma parte ha sufrido una transformación por los cambios de uso del suelo, dando origen a un paisaje en mosaico, donde $T$. reichei crece en microhábitats vulnerables (Gómez et al. 2010).

\section{Perspectivas de Conservación}

A pesar que el uso actual del sitio estudiado corresponde a un sistema agrícola que incluye praderas para el forraje y rotaciones de cultivo en hortalizas, el monitoreo permanente a los individuos de B. berteroana y T. reichei y la supervisión del manejo agropecuario son algunas de las medidas implementadas por los dueños del predio que han contribuido con la protección de la regeneración y desarrollo de ambas especies.

Al considerar los elementos de protección, origen y estado de conservación de $B$. berteroana, hacen que el sitio de estudio presente una base biológica factible para implementar algunos métodos de conservación.

La conservación de la flora amenazada requiere del apoyo y amplia información teórica. Los antecedentes permiten orientar y fundamentar acciones que aseguren prácticas e intervenciones en beneficio de una o más especies (Heywood 2014).

Tradicionalmente, la conservación ex situ se centra a nivel de especie fuera del hábitat y rango natural. Su rol es resguardar la variabilidad genética frente a una amenaza o eventual pérdida de biodiversidad. Mientras que la conservación in situ se enfoca a nivel de ecosistema y requiere necesariamente un área de tamaño adecuado y condiciones necesarias para mantener los procesos evolutivos de las poblaciones que integran una comunidad o ecosistema natural (Kanowski \& Boshier 1997). Además, Heywood (2014) indica que la conservación in situ requiere la preparación e implementación de planes detallados de conservación para especies clasificadas En Peligro o de gestión en el manejo del área/hábitat para especies Vulnerables.

Reconociendo las bondades y limitaciones de la conservación in situ y ex situ, Boshier et al. (2004) promueven el enfoque circa situm para arboles aislados o en pequeños grupos, fuera del bosque y mediante el uso (Brush 1991, Cooper et al. 1992, Kanowski \& Boshier 1997). En
México y Colombia la conservación circa situm ha sido aplicada a otras formas de crecimiento como por ejemplo a orquídeas del género Vanilla que han logrado conservarse por el uso tradicional como constituyente aromático (Herrera-Cabrera et al. 2012, Flanagan \& MosqueraEspinosa 2016 y Flanagan et al. 2018). La conservación circa situm se ha utilizado para distinguir situaciones dentro de paisajes agroforestales fuera del hábitat natural, pero dentro del ámbito geográfico natural de la especie. Durante mucho tiempo algunas especies se han mantenido, y por lo tanto conservadas, debido a su utilización por comunidades locales (Barrance et al. 2009, Herrera-Cabrera et al. 2012). En cambio, otras como Gomortega keule (Molina) Baill. y Nothofagus alessandrii Espinosa han subsistido por su capacidad de rebrotar en sistemas agrícolas o forestales, permaneciendo como remanentes del bosque nativo que las albergaba (Sepúlveda 1999, Lander et al. 2009, MuñozConcha et al. 2015, Santelices et al. 2018). Esto último podría aplicarse a los individuos de $B$. berteroana estudiados en este trabajo.

Tal como señalan Boshier et al. (2004) se cuestiona la suposición que los árboles aislados e insertos en sistemas agrícolas no cumplen un rol importante como reservorios de especies nativas o no presentan viabilidad a largo plazo y, por lo tanto, ninguna función en la conservación (Pinard et al. 2014). Si bien no existen reportes específicos de los insectos que polinizan a $B$. berteroana, San Martín et al. (2002) señalan que son visitados por dípteros e himenópteros. En este sentido, se concuerda con Boshier (2004) que los individuos aislados o en pequeños grupos de $B$. berteroana podrían desempeñar un importante rol para el flujo génico, conservación de sus recursos genéticos, tamaño poblacional y adaptabilidad al cambio climático. En este contexto, Arnold et al. (2010) sugieren que incorporar medidas de manejo y planes de gestión en sistemas agrícolas y/o forestale, implicaría: i) la conservación de genotipos no encontrados en áreas protegidas (Herrera et al. 2005, García-González et al. 2008, Venegas 2015), ii) la facilitación del flujo génico entre fragmentos (Lander et al. 2010, Guidugli et al. 2016), iii) la mantención de individuos interconectados reproductivamente a niveles necesarios para poblaciones mínimamente viables en términos genéticos y demográficos (Lander et al. 2011) y, iv) la intermediación para polinizadores y dispersores de semillas (Lander et al. 2009).

Uno de los principales desafíos que presentan algunos paisajes de producción agrícola y/o forestal es ¿Cómo compatibilizar una producción tradicional con la conservación de individuos aislados o en pequeños grupos de una especie amenazada? En este escenario, es posible distinguir dos situaciones, i) individuos localizados en la frontera del sistema agrícola y/o forestal y ii) individuos al interior del sistema. Lo habitual en el ámbito de la gestión ambiental -en un contexto productivo- sería aplicar el 
Flora y conservación de una población de Beilschmiedia berteroana: SEPÚLVEDA, C. ET AL.

principio precautorio o limitar la producción tradicional e iniciar un largo e incierto proceso de restauración ecológica. Considerando los niveles en los cuales se organiza la biodiversidad, la conservación circa situm priorizara el nivel de especie/genético por sobre el ecosistémico y, sus acciones o esfuerzos de conservación no necesariamente involucrarían una pérdida de superficie productiva, sino más bien estarían orientadas a entregar las condiciones ambientales para que ocurra la producción de flores y frutos de la(s) especie(s) a conservar.

Dado que la población estudiada de $B$. berteroana se encuentra inserta en un sistema agrícola se propone la conservación circa situm como un método complementario tanto para $B$. berteroana como $T$. reichei. Esta promoverá un mayor interés y potencial inclusión en las colecciones de semillas o bancos genéticos de campo que apoyarán la conservación ex situ (Dawson et al. 2013), integrará la producción y conservación en el mismo sitio (Barrance et al. 2009) y contribuirá con la conservación de sus recursos genéticos al incorporar individuos insertos en sistemas agrícolas o fuera del bosque (Gutiérrez et al. 2015). Sin embargo, en este escenario sería necesario investigar el comportamiento de la regeneración natural, variación y flujo genético, para comprender ¿cuál es el nivel de funcionalidad de estos individuos en este tipo de paisaje? y ¿qué medidas serán necesarias para mantener o mejorar esta funcionalidad? En esta línea se encuentran los trabajos de Herrera et al. (2005), García-González et al. (2008), Lander et al. (2009, 2010, 2011) y Muñoz-Concha \& Saud (2011) en Gomortega keule, otra especie endémica y amenazada de Chile central.

\section{AGRADECIMIENTOS}

A la Corporación Nacional Forestal (CONAF) Región del Maule por apoyar este trabajo. A los colegas de CONAF, Pablo Arriagada por la confección de los mapas y Ricardo Molina por su valioso apoyo en terreno y fotografías. A Alicia Marticorena, curadora del Herbario del Departamento de Botánica de la Universidad de Concepción (CONC), por la corroboración de las especies. A Miguel y André Pacheco por las facilidades de acceso e información del predio. A los comentarios de Franz E. Arnold, Sergio Bravo y dos revisores anónimos.

\section{REFERENCIAS}

Aguayo, M., Pauchard, A., Azócar, G., Parra, O. 2009. Cambio del uso del suelo en el centro sur de Chile a fines del siglo $\mathrm{XX}$. Entendiendo la dinámica espacial y temporal del paisaje. Revista Chilena de Historia Natural 82(3): 361-374.

Arnold, F.E., Sepúlveda, C.A., San Martín, J., Boshier, D.H., Penallillo, P., Lander, T., Garrido, P.,
Harris, S., Hawthorne, W. 2010. Propuesta de una estrategia de conservación para los bosques nativos de la sub región costera del Maule. Universidad de Oxford-Reino Unido. Universidad de TalcaChile. 96 pp. http://www.darwininitiative.org.uk/ documents/15023/20904/15-023\%20FR\%20Annex\% 20Conservation $\% 20$ Strategy $\% 20-\% 20$ Sp.pdf.

Barrance, A.J., Flores, L., Padilla, E., Gordon, J.E., SchreckenberG, K. 2003. Trees and farming in the dry zone of southern Honduras I: campesino tree husbandry practices. Agroforestry Systems 59(2): 97-106.

Barrance, A., Schreckenberg, K., Gordon, J. 2009. Conservación mediante el uso: Lecciones aprendidas en el bosque seco tropical mesoamericano. Overseas Development Institute. London. UK. $141 \mathrm{pp}$.

Benoit, I. 1989. Red List of Chilean Terrestrial Flora. CONAF, Santiago de Chile. 151 pp.

Boshier, D.H. 2004. Agroforestry systems: important components in conserving the genetic viability of native tropical tree species? In: Schroth, G., Vasconcelos, H., Harvey, C.A., Gascon, C., Fonseca, G. (eds.). Agroforestry and biodiversity conservation in tropical landscapes. Island Press, USA. pp. 290-314.

Boshier, D.H., Gordon, J.E., Barrance, A.J. 2004. Prospects for circa situm tree conservation in Mesoamerican Dry-Forest Agro-ecosystems. In: Frankie G.W., Mata, A., Vinson, S.B. (eds.). Biodiversity conservation in Costa Rica, learning the lessons in the seasonal dry forest. University of California Press. pp. 210-226.

BRUSH, S.B. 1991. A farmer-based approach to conserving crop germplasm. Economic Botany 45: 153-165.

Cabello, A., Hechenleitner, P., Gardner, F. 2006. Beilschmiedia berteroana (Gay) Kosterm Belloto del Sur, Belloto del Centro, Belloto; Avellano Familia: Lauraceae. En: Donoso, C. (ed.). Las especies arbóreas de los bosques templados de Chile y Argentina. Autoecología. Marisa Cuneo Ediciones. Valdivia. Chile. pp. 173-179.

CAIN, S. 1950. Life-forms and phytoclimate. Botanical Review (Landcaster) 16: 1-32.

Cooper, D., Vellvé, R., Hobbelink, H. (eds.). 1992. Growing diversity. Intermediate Technology Publications. 166 pp.

Dawson, I.K., Guariguata, M.R., Loo, J., Weber, J.C., Lengkeek, A., Bush, D., Cornelius J., Guarino, L., Kindt, R., Orwa, C., Russell, J., Jamnadass, J. 2013. What is the relevance of smallholder's agroforestry systems for conserving tropical tree species and genetic diversity in circa situm, in situ and ex situ settings? Biodiversity and Conservation 22(2): 301-324.

Echeverría, C., Newton, A., Lara, A., Rey-Benayas, J.M., Coomes, D.A. 2007. Impacts of forest fragmentation on species composition and forest structure in the temperate landscape of southern Chile. Global Ecology and Biogeography 16(4): 426-439.

Espinosa, M. 1941. Apuntes botánicos sobre el belloto chileno de frutos comestibles. Boletín del Museo Nacional de Historia Natural 19: 9-18.

Flanagan, N.S., Mosquera-Espinosa, A.T. 2016. An integrated strategy for the conservation and sustainable use of native Vanilla species in Colombia. Lankesteriana 16(2): 201218. 
Gayana Bot. 76(1), 2019

Flanagan, N.S., Ospina-Calderón, N., García Agapito, L.T., Mendoza, M., Mateus, H.A. 2018. A new species of Vanilla (Orchidaceae) from the North West Amazon in Colombia. Phytotaxa 364(3): 250-258.

Fuentes, N., Sánchez, P., Pauchard, A., Urrutia, J., Cavieres, L., Marticorena, A. 2014. Plantas invasoras del Centro-Sur de Chile: Una guía de Campo. Laboratorio de Invasiones Biológicas (LIB), Concepción. 276 pp.

GAJARDO, R. 1993. La vegetación natural de Chile. Clasificación y distribución geográfica. Editorial Universitaria. Santiago de Chile. 165 pp.

García-González, R., Carrasco, B., Peñailillo, P., Letelier, L., Herrera, R., Lavandero, B., Moya, M., Caligari, P.D.S. 2008. Genetic variability and structure of Gomortega keule (Molina) Baillon (Gomortegaceae) relict populations: geographical and genetic fragmentation and its implications for conservation. Botany 86: 1299-1310.

Gómez, P., Hahn S., San Martín, J., Vizcarra, G. 2010. Propagación vegetativa de Tarasa reichei Phil. mediante enraizamiento de estacas. Chloris Chilensis. Año $13-\mathrm{N}^{\circ} 1$. URL: http://www.chlorischile.cl.

Guidugli, M.C., Nazareno, A.G., Feres, J.M., Contel E.P.B., Mestriner, M.A., Alzate-Marin, A.L. 2016. Small but not isolated: a population genetic survey of the tropical tree Cariniana estrellensis (Lecythidaceae) in a highly fragmented habitat. Heredity 116: 339-347.

Gutiérrez, B., Ipinza R., Barros, S. 2015. Conservación de recursos genéticos forestales. Principios y Práctica. Instituto Forestal. $260 \mathrm{pp}$.

Hawthorne, W., Abu Juam, M. 1995. Forest Protection in Ghana. IUCN, Gland, Switzerland and Cambridge, UK. 203 pp.

Hechenleitner, P., Gardner, M.F., Thomas, P.I., Echeverria, C., Escobar, B., Martínez, C. 2005. Plantas amenazadas del Centro-Sur de Chile. Distribución, Conservación y Propagación. Universidad Austral de Chile y Real Jardín Botánico de Edimburgo. 187 pp.

Henríquez C., Azócar G., Romero, H. 2006. Monitoring and modeling the urban growth of two mid-sized Chilean cities. Habitat International 30: 945-964.

Herrera-Cabrera, B., Salazar-Rojas, V., Delgado-Alvarado, A., Campos-Contreras, J., Cervantes-Vargas, J. 2012. Use and conservation of Vanilla planifolia $\mathrm{J}$. in the Totonacapan Region, México. European Journal of Environmental Sciences 2(1): 43-50.

Herrera, I., Gonçalves, E., Pauchard, A., Bustamante, R. (eds.). 2016. Manual de Plantas Invasoras de Sudamérica. Trama Impresores S.A. Chile. 116 pp.

Herrera, R., Arias, M., Moya-León, M.A., Peñailillo, P., Wilkinson, M.J., CALigari, P.D.S. 2005. Genetic variation in a Chilean endangered endemic: Gomortega keule (Molina) Baillon. Biodiversity and Conservation 14: 2871 2881.

Heywood, V.H. 2014. An overview of in situ conservation of plant species in the Mediterranean. Flora Mediterranea 24: 5-24.

Holm, L., Pancho, J.V., Herberger, J.P., Plucknett, D.L. 1979. A geographical atlas of world weeds. J. Wiley \& Sons, New York. 391 pp.

Ibarra-Vidal, H., Sepúlveda, C.A., Saavedra, D., Maldonado, E. 2005. Propuestas de conservación de los bosques nativos en la cordillera costera del Maule y Biobío En: Sмiтh, C.,
Armesto, J., Valdovinos, C. (eds.). Historia, biodiversidad y ecología de los bosques costeros de Chile. Editorial Universitaria. Santiago, Chile, pp. 617-631.

JimÉnez-VAlverde, A., Hortal, J. 2003. Las curvas de acumulación de especies y la necesidad de evaluar la calidad de los inventarios biológicos. Revista Ibérica de Aracnología 8: 151-161.

Kanowski, P.J., Boshier, D.H. 1997. Conservation of tree genetic resources in situ. In: Maxted, N., Ford-Lloyd, B.V., Hawkes, J.G. (eds.). Plant conservation: the in situ approach. Chapman and Hall, UK. pp 207-219.

Lander, T.A., Bebber, D.P., Choy, C.T.L., Harris, S.A., Boshier, D.H. 2011. The Circe Principle Explains How ResourceRich Land Can Waylay Pollinators in Fragmented Landscapes. Current Biology 21(15): 1302-1307.

Lander, T.A., Harris, S.A., Boshier, D.H. 2009. Flower and fruit production and insect pollination of the endangered Chilean tree, Gomortega keule in native forest, exotic pine plantation and agricultural environments. Revista Chilena de Historia Natural 82: 403-412.

Lander, T.A., Harris, S.A., Boshier, D.H. 2010. Fragmented but not isolated: Contribution of single trees, small patches and long-distance pollen flow to genetic connectivity for Gomortega keule, an endangered Chilean tree. Biological Conservation 143(11): 2583-2590.

Lara, A., Araya, L., Capella, J., Fierro, M., Cavieres, A. 1989. Evaluación de la destrucción y disponibilidad de los recursos forestales nativos en la VII y VIII Región. Informe Técnico. Comité Nacional Pro Defensa Fauna y Flora (CODEFF), Santiago. 22 pp.

Lara, A., Solari, M.E., Prieto, M.R., Peña, M.P. 2012. Reconstrucción de la cobertura de la vegetación y uso de suelo hacia 1550 y sus cambios a 2007 en la ecorregión de los bosques valdivianos lluviosos de Chile ( $\left.35^{\circ}-43^{\circ} 30^{\prime} \mathrm{S}\right)$. Bosque 33(1): 13-23.

Litton, C., Díaz, J., Lara, R. 1997. Composición florística y estructura vertical de un rodal de Beilschmiedia berteroana (Gay) Kosterm. en la precordillera andina de la VII Región de Chile. Bosque 18(1): 61-72.

Marticorena, A. 2005. Malvaceae. En: Marticorena, C., Rodríguez, R. (eds.). Flora de Chile 2(3): 94-103.

Marticorena, A., Valdivieso, J.A., Baeza, C. 2007. Nuevo hallazgo de Tarasa umbellata Krapov. (Malvaceae). Gayana Botánica 64(2): 211-216.

Matteucci, S., Colma, A. 1982. Metodología para el estudio de la vegetación. Secretaría General de la OEA. Programa Regional de Desarrollo Científico y Tecnológico. Washington. $168 \mathrm{pp}$.

Matthei, O. 1995. Manual de las malezas que crecen en Chile. Alfabeta Impresores. Santiago. 545 pp.

MMA. 2017. Nómina de especies según estado conservación. Chile. Actualizado $13^{\text {er }}$ Proceso RCE. http://www.mma. gob.cl/clasificacionespecies/listado-especies-nativassegun-estado-2014.htm.

Mueller-Dombois, D., Ellenberg, H. 1974. Aims and methods of vegetation ecology. John Wiley \& Sons, New York. 547 pp.

Muñoz, C. 1973. Chile: plantas en extinción. Santiago Universitaria. $247 \mathrm{pp}$.

Muñoz-Concha, D., Farías, C., MÉndez, J. 2015. Notes on a new population of the endangered Chilean tree Gomortega 
Flora y conservación de una población de Beilschmiedia berteroana: SEPÚLVEDA, C. ET AL.

keule. New Zealand Journal of Botany 53(4): 1-7.

Muñoz-Concha, D., SAud, G. 2011. Flowering and fruiting phenology of the endangered Chilean tree Gomortega keule. New Zealand Journal of Botany 49(4): 1-6.

Myers, N., Mittermeier, R.A., Mittermeier, C.G., Da Fonseca, G.A.B., Kent, J. 2000. Biodiversity hotspots for conservation priorities. Nature 403: 853-858.

Pinard, F., JoetZjer, E., Kindt, R., Kehlenbeck, K. 2014. Are coffee agroforestry systems suitable for circa situm conservation of indigenous trees? A case study from Central Kenya. Biodiversity and Conservation 23(2): 467-495.

Ramírez, C., San Martín, C., San Martín, J., Villaseñor, R. 2004. Comparación fitosociológica de los bosques de Belloto (Beilschmiedia, Lauraceae) en Chile central. Bosque 25(1): 69-85.

Ricci, M., González, H., Cerda, R., Aguilar, A., Celis, C. 2007. Ampliación del límite norte de distribución del belloto del sur (Beilschmiedia berteroana, Lauraceae). Chloris Chilensis Año 2. Nº2. URL: http://www.chlorischile.cl.

Rodríguez, G., Rodríguez, R., Barrales, H. 1995. Plantas ornamentales chilenas. Concepción, Chile. 30 pp.

San Martín, J., SÁnchez, A. 1999. Las comunidades relictas de Gomortega keule (Gomortegaceae, Magnoliopsida) en Chile central. Anales del Jardín Botánico de Madrid 57: 317-326.

San Martín, J., Villa, A., Ramírez, C. 2002. Fenología y crecimiento vegetativo de Beilschmiedia berteroana (Gay) Kosterm. en la precordillera andina de Chile central (35 $52^{\prime}$ 'S $/ 71^{\circ} 06^{\prime} \mathrm{W}$ ). Bosque 23(1): 37-45.

Santelices, R., Espinoza, S., Cabrera, A., Magni, C. 2018. Gestión del riesgo como herramienta para la conservación de los bosques de Nothofagus alessandrii, una especie en peligro de extinción de Chile central. Interciencia 43(2): 144-150.

SEPúlvedA, C.A. 1999. Antecedentes bioecológicos y consideraciones preliminares para la conservación de Gomortega keule (Molina) Baillon bajo la cobertura de Pinus radiata D. Don, en el fundo Tregualemu (Pelluhue), Región del Maule, Chile. Programa Forestal. CODEFF. 20 pp.

Tapia, M., Finot, V., Merino, V. 2000. Plantas tóxicas de la VIII Región. Descripción botánica, principios tóxicos, mecanismos de acción y sintomatología en animales afectados. Primera Edición. Universidad de Concepción. $81 \mathrm{pp}$.

Troncoso, A. 1988. Flora I. En: Meléndez, T. (ed.). Enciclopedia temática de Chile Tomo 12. Sociedad Editora Revista Ercilla. Chile. 60 pp.

VAn Der MaArel, E. 1979. Transformation of cover-abundance values in phytosociology and its efects on community similarity. Division of Geobotany, Nijmegen, The Netherlands. Vegetatio 39(2): 97-114.

Venegas, C.F. 2015. Variabilidad genética en sub-poblaciones de Pitavia punctata Mol. especie endémica amenazada del sur de Chile. Tesis Ingeniería en Biotecnología Vegetal, Facultad de Ciencias Forestales, Universidad de Concepción. Concepción, Chile. 55 pp.

Walter, K.S., Gillett, H.J. 1998. 1997 IUCN Red List of Threatened Plants. IUCN - The World Conservation Union. Gland, Switzerland and Cambridge, UK. 936 pp.

Recibido: 27.07 .2018

Aceptado: 17.10 .2018 